\title{
Role of elasticity forces in thermodynamics of intercalation compounds: Self-consistent mean-field theory and Monte Carlo simulations
}

\author{
V. I. Kalikmanov a) and S. W. de Leeuw \\ Department of Applied Physics, Computational Physics Section, University of Delft, Lorentzweg 1, \\ 2628 CJ Delft, The Netherlands
}

(Received 11 October 2001; accepted 27 November 2001)

\begin{abstract}
We propose a self-consistent mean-field lattice-gas theory of intercalation compounds based on effective interactions between interstitials in the presence of the host atoms. In addition to short-range screened Coulomb repulsions, usually discussed in the lattice gas models, the present theory takes into account long-range effective attractions between intercalants due to elasticity of the host matrix. The mean-field phase diagram in the space of interaction parameters contains the domains of first- and second-order transitions of the order-disorder type, separated by a tricritical line, and the domain of the first-order transition of the gas-liquid-type separated from the homogeneous state by a critical line. Theoretical predictions are shown to be in qualitative agreement with the grand canonical Monte Carlo simulations. The peculiarities of the phase diagram give an insight into different types of behavior of the open circuit voltage observed in rechargeable batteries, in which an intercalation compound is used as an electrode material. (C) 2002 American Institute of Physics. [DOI: 10.1063/1.1436472]
\end{abstract}

\section{INTRODUCTION}

An intercalation compound represents a host matrix with a number of guest atoms occupying interstitial sites. In alkali metal intercalation compounds-an example is $\mathrm{Li}_{x} \mathrm{MO}_{2}$ where lithium is intercalated into a metal(M)-oxide matrix - the guest is fully ionized and donates its $s$-electron to the host's energy bands. These materials attract considerable attention because of their potential use as high-density battery electrodes (see e.g. Refs. 1, 2, and references therein).

Absorption of ions in the course of intercalation is frequently accompanied by structural transformations of the host matrix, ${ }^{3,4}$ suggesting the importance of elasticity effects. This statement is supported by x-ray measurements ${ }^{5}$ and $a b$ initio calculations. ${ }^{6}$ A statistical mechanical description of intercalation compounds is usually based on lattice gas models with the total neglect of elasticity effects. In the present paper we take into account the elastic properties of the host material in a simplified manner using the self-consistent mean-field approximation (Sec. II). The model gives rise to the mean-field phase diagram. Analyzing it we identify various types of phase transitions (Sec. III)-order-disorder, gas-liquid-which occur in the compound at a certain range of parameters (temperature, elasticity coupling). To test the theory we performed Monte Carlo simulations in the grand canonical ensemble (Sec. IV). The phase diagram qualitatively explains the peculiarities of the experimentally observed open circuit voltage of intercalation cells in which an intercalation compound is used as an electrode material. An application of the theory for the open circuit voltage calculations and comparison with experiment is presented in Sec. V.

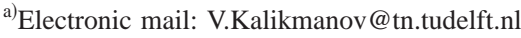

\section{MODEL}

We treat an intercalation compound as a one-component lattice gas of interstitials interacting via effective potentials mediated by the host matrix. We assume that the interstitial lattice is bichromatic with a coordination number $q$ and a total number of sites $N$. All sites are assumed to be equivalent. The basic effective interactions are due to (i) electrostatic forces and (ii) elasticity of the host material. We discuss them below in more detail.

Coulomb repulsion between intercalated ions is screened by the negative charge of the host electrons. In typical compounds the large electron concentration of the host results in a small screening length - of the order of a lattice spacing. It is therefore plausible to assume that repulsions are between nearest neighbors, the pair interaction energy $u$ $\sim 30-70 \mathrm{meV} .{ }^{7}$ With each site $i$ we associate a variable $p_{i}$, which is unity if the site is occupied and zero otherwise. The repulsive energy of an arbitrary configuration is

$$
E_{0}\left(p_{1}, \ldots, p_{N}\right)=u \sum_{(i, j)_{n n}} p_{i} p_{j}
$$

where $(i, j)_{n n}$ denotes summation over nearest neighbors with each pair counted only once.

The host lattice is deformable and absorption of guest ions induces a long-range strain field in it. The elastic energy of the strained crystal is equivalent to an effective interaction, $u_{\mathrm{el}, i j}$, between intercalants mediated by the host. Its strength is independent of the size of the sample but depends on elastic conditions imposed on its boundary. ${ }^{8}$ In the following, ${ }^{9}$ we divide $u_{\mathrm{el}, i j}$ into two parts,

$$
u_{\mathrm{el}, i j}=u_{\mathrm{el}}^{\infty}\left(r_{i j}\right)+u_{\mathrm{el}}^{s}\left(\mathbf{r}_{1}, \mathbf{r}_{2}\right),
$$

where $r_{i j}=\left|\mathbf{r}_{1}-\mathbf{r}_{2}\right|$ is the separation between intercalants. The first term is the interaction in an infinite host medium and the second arises from image forces which are required 
to satisfy the boundary conditions imposed on the surface of the sample. In our investigation of the elasticity effects we will resort to a simplified description based on the following approximations. (a) The host material is assumed to be elastically isotropic, which means that its elasticity properties are described by the two scalar parameters (e.g., the Young modulus $Y$ and the Poisson ratio $\nu$ ). (b) The intercalation process causes equal extension of the host in all three dimensions. (c) The surface of the sample is free.

In elasticity theory the effect of an intercalated particle on a host can be adequately described by the "force dipole tensor" 10 which is a first moment tensor of the body forces f in the elastic medium,

$$
P_{i j}=\int \mathrm{d} \mathbf{r} x_{i} f_{j}
$$

where $x_{i}$ and $f_{j}$ are Cartesian coordinates of $\mathbf{r}$ and $\mathbf{f}$. Assumptions (a) and (b) lead to a particularly simple form of $\mathbf{P}$,

$$
P_{i j}=P_{0} \delta_{i j},
$$

where $\delta_{i j}$ is the Kronecker delta. The bulk contribution in Eq. (2) can be expressed in terms of the diagonal components of $\mathbf{P}$ and the infinite medium Green's function $G_{i k}(\mathbf{r}) .^{9,11}$ If a particle is intercalated in the origin, then for another particle located at the point $\mathbf{r}$ the bulk interaction energy with the first one is given by

$$
u_{\mathrm{el}}^{\infty}(r)=P_{0}^{2} \sum_{i=1}^{3} \sum_{j=1}^{3} \frac{\partial G_{i k}}{\partial x_{i} x_{k}} .
$$

For an isotropic medium [assumption (a)] Green's function reads $^{12}$

$$
G_{i k}=a \frac{x_{i} x_{k}}{r^{3}}+b \frac{1}{r} \delta_{i k},
$$

with the coefficients $a(Y, \nu)$ and $b(Y, \nu)$ depending on the elastic constants of the host material. From Eqs. (3) and (4) we find $u_{\mathrm{el}}^{\infty}(r) \equiv 0$. Thus, under assumptions (a) and (b) the bulk contribution to the elastic interaction vanishes. The surface contribution depends on the imposed boundary conditions. For a completely free sample [assumption (c)] it is known to be attractive and infinitely long-range. ${ }^{11,13}$ [Note that for a perfectly clamped surface, whose volume is kept constant (no expansion), the effective elastic interaction can be repulsive; in practice, however, it is difficult to clamp the sample without hindering the intercalation process.] Summarizing, we can write

$$
u_{\mathrm{el}, i j}=-A_{\mathrm{el}} p_{i} p_{j} .
$$

Thus, the effective elastic interactions in our model reduce to the infinitely long-range attractions. The elastic coupling $A_{\mathrm{el}}$ can be expressed in terms of $Y, \nu$, and $P_{0}$. However, in the present model, we consider $A_{\mathrm{el}}$ a fitting parameter for comparison with experimental data.

The elastic interaction energy of an arbitrary configuration of intercalants reads

$$
E_{\mathrm{el}}\left(p_{1}, \ldots, p_{N}\right)=-A_{\mathrm{el}} \frac{1}{2 N} \sum_{i, j} p_{i} p_{j}
$$

In the spirit of a mean-field approach we introduce the average density of intercalants $x=\left\langle p_{i}\right\rangle$ and approximate (6) as

$$
E_{\mathrm{el}}\left(p_{1}, \ldots, p_{N}\right) \approx-A_{\mathrm{el}} x \sum_{i} p_{i}+\frac{1}{2} A_{\mathrm{el}} x^{2} N
$$

Summarizing, the full Hamiltonian is

$$
E\left(p_{1}, \ldots, p_{N}\right)=u \sum_{(i, j)_{n n}} p_{i} p_{j}-A_{\mathrm{el}} x \sum_{i=1}^{N} p_{i}+\frac{1}{2} A_{\mathrm{el}} x^{2} N
$$

Consider the grand canonical $(\mu, N, T)$ ensemble, where $\mu$ is the chemical potential of an intercalant, and $T$ is the temperature. The number of occupied sites is not fixed implying that the average occupation $x$ depends on $\mu$ and $T$. The grand partition function of the lattice gas with the Hamiltonian (8) is

$$
\begin{aligned}
\Xi(\mu, N, T)= & \exp \left[-\frac{1}{2} \beta A_{\mathrm{el}} x^{2} N\right] \\
& \times \sum_{\{p\}} \exp \left\{\left[\beta \sum_{i=1}^{N}\left(\mu+A_{\mathrm{el}} x\right) p_{i}\right] \exp \left[-\beta E_{0}\right]\right\},
\end{aligned}
$$

where the summation in the outer sum is over all configurations of intercalants, $\beta=1 /\left(k_{B} T\right), k_{\mathrm{B}}$ is the Boltzmann constant. We stress that $x$ enters $\Xi$ in a self-consistent way; $x$ $=x(\mu, T)$. An important observation is that elastic interactions in our model give rise to the renormalization of the chemical potential,

$$
\mu \rightarrow \mu+A_{\mathrm{el}} x(\mu, T),
$$

compared to the system with pure repulsions (the latter case refers to the topotactic intercalation ${ }^{14}$ ).

At this stage it is convenient to formulate an equivalent spin system: a fully occupied lattice of $N$ spins $s_{i}= \pm 1$ defined through the relationship $s_{i}=2 p_{i}-1$. Using the standard technique $^{15}$ we find for the grand potential of the lattice gas $\Omega=-k_{\mathrm{B}} T \ln \Xi$,

$$
\begin{aligned}
\Omega(\mu, N, T)= & -N\left[\frac{\mu}{2}-\frac{q u}{8}+\frac{A_{\mathrm{el}}}{4}(1+m)\right. \\
& \left.-\frac{A_{\mathrm{el}}}{8}(1+m)^{2}\right]+\mathcal{F}_{\mathrm{Is}},
\end{aligned}
$$

where $\mathcal{F}_{\text {Is }}$ is the Helmholtz free energy of the antiferromagnetic Ising model with a coupling energy $u / 4$ in an external field

$$
H=\frac{\mu}{2}-\frac{q u}{4}+\frac{A_{\mathrm{el}}}{4}(1+m)
$$

where $m=\left\langle s_{i}\right\rangle=2 x-1$ is the average magnetization per spin. The "Ising Hamiltonian" reads

$$
\mathcal{H}_{\mathrm{Is}}=\frac{u}{4} \sum_{(i, j)_{n n}} s_{i} s_{j}-H \sum_{i} s_{i}
$$




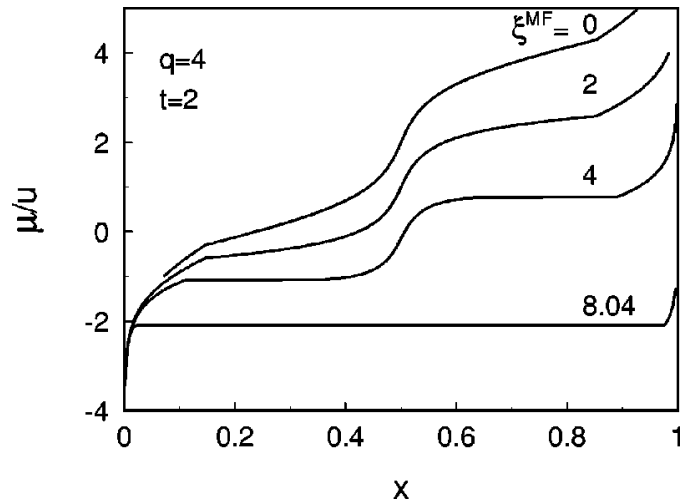

FIG. 1. Chemical potential vs concentration for the lattice with the coordination number $q=4$ at the temperature $t=2$. The mean-field critical temperature $t_{c}^{\mathrm{MF}}=q=4$. Curves are labeled with the value of $\xi^{\mathrm{MF}} ; \xi^{\mathrm{MF}}=0$ corresponds to the absence of attractions.

The self-consistent nature of the original lattice gas is inherited by the spin system by virtue of the external field dependence on the magnetization [Eq. (10)] which itself is a function of the field, $m=m(H, T)$.

The magnetization is an odd function of $H$ implying that $x(-H)=1-x(H)$. In turn for each $x$ there is a unique value of $\mu$; thus, using Eq. (10) we find a general property of the model reflecting the equivalence of sites (a "particle-hole symmetry"),

$$
\mu(x)+\mu(1-x)=\left(q-\xi^{\mathrm{MF}}\right) u \text { for all } t .
$$

In particular, at the half-filling,

$$
\mu\left(x=\frac{1}{2}\right)=\frac{\left(q-\xi^{\mathrm{MF}}\right) u}{2} .
$$

Where we introduced a reduced temperature $t=4 k_{B} T /$ $u$, and a reduced elastic coupling,

$$
\xi^{\mathrm{MF}}=\frac{A_{\mathrm{el}}}{u}
$$

representing a relative strength of the elastic interactions with respect to (electrostatic) repulsions. Recalling that the interstitial lattice is bichromatic and applying the mean-field approximation, we obtain from Eq. (11) the coupled selfconsistent mean-field equations for magnetizations $m_{1}$ and $m_{2}$ of the sublattices,

$$
\begin{aligned}
& m_{1}+\tanh \left[h+\frac{\xi^{\mathrm{MF}}}{2 t} m_{1}-\frac{1}{2 t}\left(2 q-\xi^{\mathrm{MF}}\right) m_{2}\right], \\
& m_{2}=\tanh \left[h+\frac{\xi^{\mathrm{MF}}}{2 t} m_{2}-\frac{1}{2 t}\left(2 q-\xi^{\mathrm{MF}}\right) m_{1}\right],
\end{aligned}
$$

where

$$
h=\frac{2 \mu}{u t}+\gamma, \quad \gamma=\frac{\xi^{\mathrm{MF}}-q}{t} .
$$

The magnetization per spin for the entire lattice is $m=\left(m_{1}\right.$ $\left.+m_{2}\right) / 2$.

In the general case (i.e., when $m_{1} \neq m_{2}$ ) the equations must be solved numerically. Figure 1 illustrates the behavior of $\mu(x)$ resulting from Eqs. (14) and (15) for $q=4$ and $t$

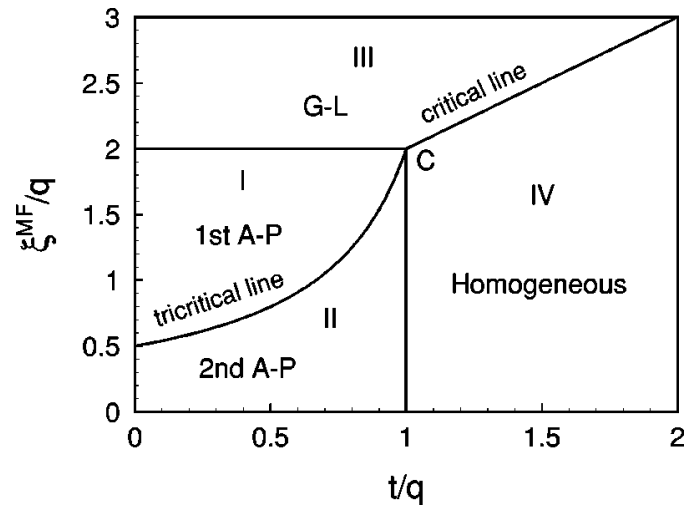

FIG. 2. Phase diagram of an intercalation compound. I, domain of 1st order antiferromagnetic-paramagnetic (A-P) transitions; II, domain of second order A-P transitions; III, domain of gas-liquid (G-L) transitions; IV, homogeneous state.

$=2$. Curves are labeled by the values of the elastic coupling. One can see that a shape of a curve strongly depends on the value of $\xi^{\mathrm{MF}}$. All the curves shown in Fig. 1 contain singularity points—kinks and/or plateaus—signaling about phase transitions of different types.

\section{PHASE DIAGRAM}

Equations (14)-(15) give rise to the mean-field phase diagram. Let us first discuss some of its features qualitatively. At low temperatures in the absence of elastic forces $\left(\xi^{\mathrm{MF}}=0\right)$ the short-range repulsions result in the orderdisorder (antiferromagnetic-paramagnetic) continuous phase transition ${ }^{14}$ at a certain critical value of the chemical potential; the order parameter being the staggered magnetization,

$$
\phi=\left(m_{1}-m_{2}\right) / 2 .
$$

Note, that in the original lattice-gas formulation $\phi=x_{1}$ $-x_{2}$, where $x_{1}$ and $x_{2}$ are the average occupations of the sublattices, the ordered state corresponds to the preferential occupation of one of the sublattices.

If attractive (elastic) interactions are "switched on" $\left(\xi^{\mathrm{MF}}>0\right),{ }^{16}$ they compete with repulsions and beyond a certain value of $\xi^{\mathrm{MF}}$ this transition is driven first order. In this case $\phi$ jumps at the transition point (i.e., at some $\mu$ ) from zero to a finite value. An infinitesimally small jump corresponds to the tricritical point, where both types of transition meat each other. If attractions are sufficiently strong the antiferromagnetic ordering does not occur at all being replaced by the ferromagnetic ordering making no distinction between sublattices. In this case at low temperatures a first-order transition of the gas-liquid type takes place.

Figure 2 shows the phase diagram of an intercalation compound in the space of parameters $\left(t / q, \xi^{\mathrm{MF}} / q\right)$. In the low-temperature region $0 \leqslant t / q \leqslant 1$ domains I and II correspond to the antiferromagnetic-paramagnetic (A-P) transition, which is first-order in I and continuous in II. By this we mean that, if we fix an arbitrary point $\left(t / q, \xi^{\mathrm{MF}} / q\right)$ in I or II and study the behavior of the system at different values of $\mu$, then at a certain $\mu_{c}$ the system undergoes the A-P phase transition (either first-order or continuous); the latter results in the presence of singularity points on the $x(\mu)$ curve. 
In the symmetric phase $m_{1}=m_{2}=m$ and Eqs. (14) and (15) reduce to the single equation,

$$
m=\tanh \left[h+\left(\frac{\xi^{\mathrm{MF}}-q}{t}\right) m\right]
$$

yielding the analytical solution, which in the original lattice gas variables reads

$$
\mu^{\mathrm{symm}}(x)=\left(q-\xi^{\mathrm{MF}}\right) u x+k_{B} T \ln \left(\frac{x}{1-x}\right) .
$$

It gives the free energy cost for the insertion of an intercalant into the host matrix. The second term is the entropic contribution. The first term is caused by interactions; depending on the strength of elastic forces it can be both positive and negative. In the nonsymmetric phase Eqs. (14)-(15) must be solved numerically.

The boundary between the domains I and II is given by the line of tricritical points (tricritical line). In numerical calculations it is difficult to determine accurately the location of the tricritical point $\xi_{3 c}^{\mathrm{MF}}$, since the first-order transition in its vicinity is very weak. To find $\xi_{3 c}^{\mathrm{MF}}$ we apply the molecular field theory. Let us write the magnetization of sublattices in the vicinity of the tricritical point as $m_{1}=m+\phi, m_{2}=m$ $-\phi$. Equations (14)-(15) can be presented as

$$
\begin{aligned}
& 2 m=\tanh (y+r \phi)+\tanh (y-r \phi), \\
& 2 \phi=\tanh (y+r \phi)-\tanh (y-r \phi),
\end{aligned}
$$

where $r \equiv q / t$ and $y \equiv h+\gamma m$. Note that both $y$ and $m$ depend on $\phi$. Using the smallness of $\phi$ near the tricritical point and expanding all quantities in powers of $\phi$ to the third order, we derive a self-consistent equation

$$
\phi=b_{1} \phi+b_{3} \phi^{3}+O\left(\phi^{5}\right)
$$

with the coefficients

$$
\begin{aligned}
& b_{1}=\frac{r}{\cosh ^{2} y_{0}}, \\
& b_{3}=\left[\frac{2 \sinh ^{2} y_{0}-1}{3 \cosh ^{4} y_{0}}-\frac{2 \gamma a_{0} \tanh y_{0}}{\cosh ^{2} y_{0}}\right] r^{3},
\end{aligned}
$$

where

$$
y_{0}=\operatorname{arctanh} m_{0}, \quad a_{0}=\frac{m_{0}\left(1-m_{0}^{2}\right)}{\left(1-m_{0}^{2}\right) \gamma-1},
$$

and $m_{0}$ satisfies the self-consistency relation,

$$
m_{0}=\tanh \left(h+\gamma m_{0}\right) .
$$

The tricritical point is given by the conditions, $b_{1}=1$ and $b_{3}=0$. The first equality using Eq. (20) yields

$$
m_{0}= \pm \sqrt{1-\frac{t}{q}}
$$

imposing the standard mean-field temperature requirement, $t<t_{c}^{\mathrm{MF}}=q$. The second equality combined with Eqs. (21) and (22) leads to the value of $\xi^{\mathrm{MF}}$ at the tricritical point,

$$
\frac{\xi_{3 c}^{\mathrm{MF}}}{q}=f\left(\frac{t}{q}\right), \quad f(z) \equiv \frac{2}{4-3 z}, \quad 0 \leqslant z \leqslant 1 .
$$

If elastic interactions are sufficiently strong, $\xi^{\mathrm{MF}} / q>2$, the antiferromagnetic state is unstable for all $\mu$ while the stable ordered phase is ferromagnetic with $m_{1}=m_{2}=m$ satisfying $m=\tanh (h+\gamma m)$. The first-order transition of the gas-liquid type $(\mathrm{G}-\mathrm{L})$ with the jump in the magnetization $m \rightarrow-m$ takes place in the zero field $(h=0)$ when $\gamma>1$ (domain III in Fig. 2). Thus, from Eq. (16) the value of $\mu$ at the $\mathrm{G}-\mathrm{L}$ transition point,

$$
\mu_{\mathrm{G}-\mathrm{L}}=\frac{\left(q-\xi^{\mathrm{MF}}\right) u}{2}
$$

is independent of the temperature.

If one continued the critical line $\gamma=1$ into the lowtemperature region $t / q<1$, one would observe that a part of the domain I above this line is also a candidate for the $\mathrm{G}-\mathrm{L}$ transition. To decide which of the two transitions, A-P or $\mathrm{G}-\mathrm{L}$, is realized one has to determine the thermodynamically stable phase. A straightforward way to do this is to compare the energies per spin of the antiferromagnetic and ferromagnetic states at $t=0$. From Eqs. (10) to (13),

$$
\begin{aligned}
e=\frac{\mathcal{H}_{\mathrm{Is}}}{N}= & \frac{u}{4 N} \sum_{(i, j)} s_{i} s_{j}-\frac{H}{N} \sum_{i} s_{i}, \\
& \text { where } H=\frac{\mu}{2}-\frac{q u}{4}+\frac{\xi^{\mathrm{MF}} u}{4}(1+m) .
\end{aligned}
$$

In the antiferromagnetic state at $t=0$ all spins of one of the sublattice are "up," and those of the other are "down;" $s_{i}^{(1)}=m_{1}=1, s_{i}^{(2)}=m_{2}=-1$ for all $i$, implying that

$$
H=H_{a}=\frac{\mu}{2}-\frac{q u}{4}+\frac{\xi^{\mathrm{MF}} u}{4} \text {. }
$$

The energies of the sublattices are

$$
e_{a}^{(1)}=-\frac{q u}{4}-H_{a}, \quad e_{a}^{(2)}=-\frac{q u}{4}+H_{a},
$$

yielding the energy per spin of the entire system,

$$
e=e_{a}=\frac{e_{a}^{(1)}+e_{a}^{(2)}}{2}=-\frac{q u}{4} .
$$

In the ferromagnetic state at $t=0$, all spins have the same value, $s_{i}^{(1)}=s_{i}^{(2)}=m$ and $m$ is either +1 or -1 . For $m=+1$,

$$
\begin{aligned}
& H=H_{f, \uparrow}=\frac{\mu}{2}-\frac{q u}{4}+\frac{\xi^{\mathrm{MF}} u}{2}, \\
& e=e_{f, \uparrow}=\frac{q u}{4}-H_{f, \uparrow}=-\frac{\mu}{2}+\left(\frac{q-\xi^{\mathrm{MF}}}{2}\right) u .
\end{aligned}
$$

For $m=-1$,

$$
H=H_{f, \downarrow}=\frac{\mu}{2}-\frac{q u}{4}, \quad e=e_{f, \downarrow}=\frac{q u}{4}+H_{f, \downarrow}=\frac{\mu}{2} .
$$

Note that from the three quantities $e_{f, \uparrow}, e_{f, \downarrow}$, and $e_{a}$ only $e_{f, \uparrow}$ depends on the elastic coupling. Figure 3 compares $e_{a}$ with $e_{f, \uparrow}$ and $e_{f, \downarrow}$. If $\xi^{\mathrm{MF}} / q<2$ the antiferromagnetic state is stable in a certain range of $\mu$. For illustration we show in Fig. 3 the dashed line corresponding to $e_{f, \uparrow}$ for $\xi^{\mathrm{MF}} / q=1$; 


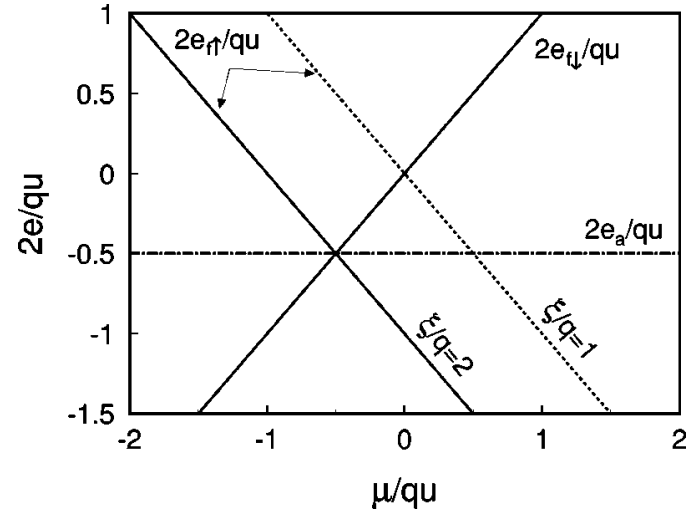

FIG. 3. Energy per spin of the antiferromagnetic $\left(e_{a}\right)$ and ferromagnetic $\left(e_{f \uparrow}\right.$ and $\left.e_{f \downarrow}\right)$ state at $t=0$.

one can see that the antiferromagnetic state is stable for $-1 / 2<\mu /(q u)<1 / 2$. When $\xi^{\mathrm{MF}} / q$ exceeds 2 , the antiferromagnetic ordering becomes energetically unfavorable. Thus, inside the entire domain I of the phase diagram the first-order A-P transition takes place.

In the high-temperature region $t / q>1$ the system can either undergo a $\mathrm{G}-\mathrm{L}$ transition or be in a homogeneous state. The boundary between III and IV is given by the line of critical points (critical line) $\gamma=1$,

$$
\frac{\xi^{\mathrm{MF}}}{q}=\frac{t}{q}+1
$$

The $\mathrm{G}-\mathrm{L}$ transition in III results in a broad plateau of $\mu(x)$ symmetric about $x=1 / 2$. The value $\mu$ at coexistence is given by Eq. (24).

\section{MONTE CARLO SIMULATIONS}

The phase diagram discussed in Sec. III is by construction mean-field. As such it is expected to be qualitatively correct, though quantitative differences with the "exact solution" are unavoidable (except for the zero temperature where the mean-field description becomes exact). The meanfield phase diagram does not depend on the dimensionality $D$ of the lattice; at the same time the "exact solution" must possess a $D$-dependence. In order to verify predictions of the theory we performed Monte Carlo (MC) simulations (which can be viewed as the "exact solution") of the lattice gas in the grand canonical $(\mu, N, T)$ ensemble with the Hamiltonian,

$$
\mathcal{H}=u \sum_{(i, j)_{n n}} p_{i} p_{j}-A_{\mathrm{el}} \frac{1}{2 N} \sum_{i, j} p_{i} p_{j} .
$$

Simulations were performed for the 2D square lattice ( $q$ $=4$ ) containing $N=900$ sites with periodic boundary conditions and $10^{3} \mathrm{MC}$ steps per lattice site. Some of the results are presented in Figs. 4 and 5 showing the plots $x(\mu)$ and $\phi(\mu)$, respectively, for the temperature $t=1.63$ and the two values of the elastic coupling, $\xi^{\mathrm{MF}}=2$ and $\xi^{\mathrm{MF}}=6$.

A general property of the model with the Hamiltonian (26), manifesting the particle-hole symmetry, is the value of $\mu$ at a half-filling given by Eq. (12). This result is independent of the approximation (in this case, mean-field) used to

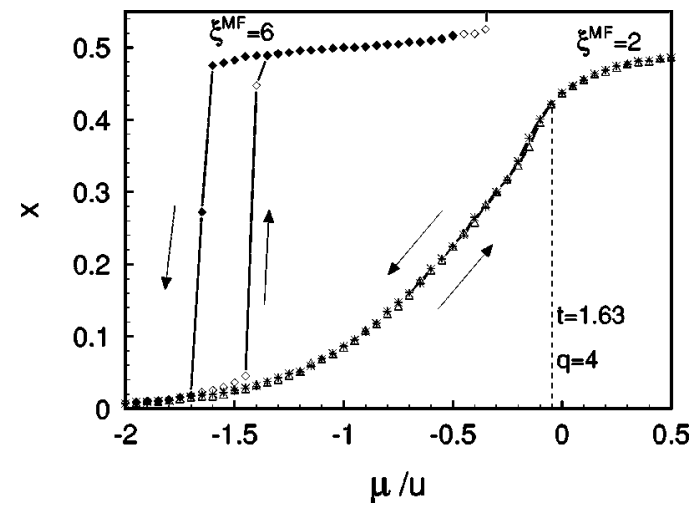

FIG. 4. Density vs chemical potential for the square lattice at $t=1.63$ : Monte Carlo simulations. Curves are labeled by the value of $\xi^{\mathrm{MF}}$. Arrows show the direction of changing $\mu$ in simulations. Hysteresis observed for $\xi^{\mathrm{MF}}=6$ manifests a first-order transition.

solve the model. This should also hold for MC and can serve as a test for $\mathrm{MC}$ results. For the square lattice and $\xi^{\mathrm{MF}}=6$, Eq. (12) predicts

$$
\left(\frac{\mu}{u}\right)_{x=1 / 2}^{\text {theory }}=-1 \text {. }
$$

From the simulations we obtain

$$
\left(\frac{\mu}{u}\right)_{x=1 / 2}^{\mathrm{MC}}=-0.95 \div-1 .
$$

For $\xi^{\mathrm{MF}}=2$,

$$
\left(\frac{\mu}{u}\right)_{x=1 / 2}^{\text {theory }}=1, \quad\left(\frac{\mu}{u}\right)_{x=1 / 2}^{\mathrm{MC}}=0.95 \div 1 .
$$

This comparison shows that MC results satisfy the particlehole symmetry.

For $\xi^{\mathrm{MF}}=2$ the point $\left(t / q, \xi^{\mathrm{MF}} / q\right)=(0.41,0.5)$ falls in the domain II of the phase diagram of Fig. 2 predicting the second-order transition of the order-disorder-type at some critical value of $\mu$. For $\xi^{\mathrm{MF}}=6$ the point $\left(t / q, \xi^{\mathrm{MF}} / q\right)$ $=(0.41,1.5)$ falls in the domain $\mathrm{I}$, where this transition is first-order. A clear indication of a first-order transition ob-

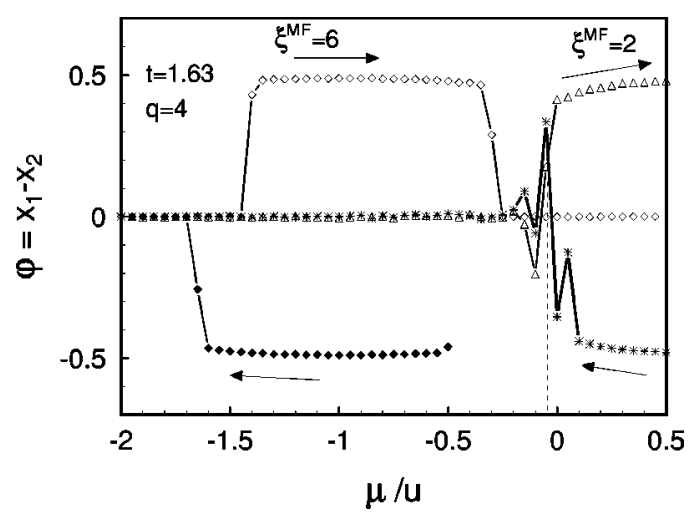

FIG. 5. Order parameter vs chemical potential for the square lattice at $t$ =1.63: Monte Carlo simulations. Notations are the same as in Fig. 4 . 
served in simulations is a hysteresis on the $x(\mu)$ curve for $\xi^{\mathrm{MF}}=6$ (Fig. 4) and $\phi(\mu)$ curve (Fig. 5), where the arrows show the direction of changing $\mu$.

For $\xi^{\mathrm{MF}}=2$ the "forward" and "backward" curves coincide to within the error bars; the location of the transition point $\mu_{c} / u \approx-0.05$ (vertical dashed line) is determined from the maximum of the staggered susceptibility monitored during the simulation runs,

$$
\chi_{s}=N\left[\left\langle x_{s}^{2}\right\rangle-\left(\left\langle x_{s}\right\rangle\right)^{2}\right], \quad x_{s}=\frac{n_{1}-n_{2}}{N},
$$

where $n_{1}$ and $n_{2}$ are occupations of the sublattices. The location of the transition point for $\xi^{\mathrm{MF}}=6$ (first-order phase transition) can be found from the Maxwell construction.

The mean-field tricritical point for the square lattice at $t=1.63$ is from Eq. (23): $\xi_{3 c}^{\mathrm{MF}} \approx 2.89$. Our MC calculations predict the higher value: $\xi_{3 c}^{\mathrm{MC}} \approx 5.2 \div 5.3$. The boundary between the $\mathrm{G}-\mathrm{L}$ and $\mathrm{A}-\mathrm{P}$ transition is found to be at $\xi^{\mathrm{MF}}$ $\approx 8.25$, which is close to the mean-field prediction $\xi^{\mathrm{MF}}=8$ (the horizontal line in Fig. 2).

\section{OPEN CIRCUIT VOLTAGE}

The phase behavior of an intercalation compound studied in the previous sections is intimately related to the open circuit voltage $(\mathrm{OCV})$ of batteries in which an intercalation compound is used as an electrode material. During a discharge cycle guest ions (e.g., $\mathrm{Li}^{+}$) diffuse through the electrolyte from anode to cathode and are inserted interstitially in the host lattice donating the equal number of electrons to the host's energy bands; the counterion transport through electrolyte is completely prohibited. Ions accumulated in the host matrix during the discharge cycle are removed from it upon charging (in an ideal cell reversibly). The cell voltage $V$ is found from the energy balance, ${ }^{1}$

$$
V(x)=-\frac{1}{e_{e}} \mu(x)+V_{0},
$$

where $e_{e}$ is the electron charge and $V_{0}$ sets the voltage scale. Thus, measuring at equilibrium the cell voltage versus charge passed between the electrodes is equivalent to measuring the chemical potential as a function of the concentration of intercalants.

In fact the chemical potential in Eq. (29) should be a sum of contributions from ions and electrons,

$$
\mu=\mu_{\text {ion }}+\mu_{\text {electron }}
$$

for a singly charged ion. Although this separation is not unique because of strong interactions between ions and electrons, in metals it is often possible to arrange the interaction terms so that $\mu_{\text {electron }}$ is constant. ${ }^{1}$ In what follows we include this constant into $V_{0}$ and consider $\mu(x)$ in Eq. (29) to be the ionic contribution $\mu_{\text {ion }}(x)$.

Figure 6 shows a comparison of the theoretical OCV with the experimental results for spinel $\mathrm{Li}_{x} \mathrm{Mn}_{2} \mathrm{O}_{4}$ at 300 $\mathrm{K},{ }^{17}$ where Li ions occupy a 3D diamond lattice with $q=4 .{ }^{18}$ Parameters of the model are found from the fit to experiment

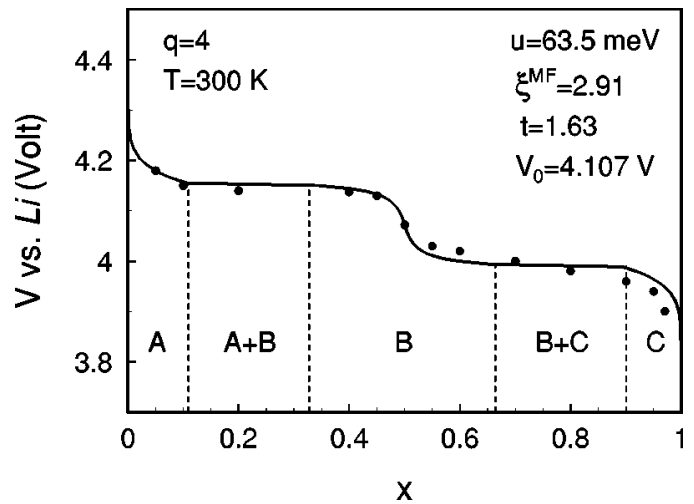

FIG. 6. OCV for $\mathrm{Li}_{x} \mathrm{Mn}_{2} \mathrm{O}_{4}$ at $300 \mathrm{~K}$. Circles, experiment of Liu et al. (Ref. 17); solid line, theory.

at $x=1 / 2$, where $\mu$ is given by Eq. (12), and at some small $x=\delta \ll 1$ corresponding to a disordered state, for which from Eq. (17),

$$
\mu(\delta)=\left(q-\xi^{\mathrm{MF}}\right) u \delta+k_{B} T \ln \frac{\delta}{1-\delta} .
$$

Using as an estimate $u \approx 63.5 \mathrm{meV}$ (see, e.g., Ref. 7) we obtain $\xi^{\mathrm{MF}}=2.91, V_{0}=4.107 \mathrm{~V}$ yielding $t=1.63, \xi^{\mathrm{MF}} / q$ $=0.728$. These values of parameters correspond to the elastic coupling energy $A_{\mathrm{el}}=u \xi^{\mathrm{MF}} \approx 0.182 \mathrm{eV}$. From Eq. (23) we find the mean-field tricritical point $\xi_{3 c}^{\mathrm{MF}} / q=0.722$ indicating that the system falls into the domain I of the phase diagram and therefore at the certain values of $\mu$ undergoes a firstorder A-P transition resulting in the two-plateau profile of the OCV.

Our theory suggests that the intercalation of lithium into the spinel structure may be divided into several steps. From the fully charged state when $x=0$ the host matrix accommodates $\mathrm{Li}$ ions as a single phase, $A$, up to $x=0.107$, this stage corresponds to a sharp voltage drop. Then, the first-order transition takes place leading to the formation of the twosublattice structures. For $0.107<x<0.345 \mathrm{Li}$ ions occupy the sites belonging to one of the sublattices in a way that gives rise to a coexistence, $A+B$, between a lithium-poor phase, $A$, and a lithium-rich phase, $B$. This leads to a flat plateau at a potential $4.15 \mathrm{~V}$. At $x=0.345$ the first sublattice consists solely of $B$ and intercalation proceeds until the first sublattice becomes fully occupied (at $x=1 / 2$ ) after which occupation of the second sublattice begins causing a substantial voltage drop $(\sim 100 \mathrm{mV})$. Intercalation into the second sublattice shows the same features as intercalation into the first one. Finally, at $x=0.893$ the first order transition takes place and lithium ions fill the rest unoccupied sites randomly.

Although the agreement between theory and experiment is quite good, one can notice that while the theoretical curve is symmetric about $x=1 / 2$ (due to the particle-hole symmetry of the model), the experimental data are not. A possible qualitative explanation to this discrepancy is that the theory does not take into account the permselectivity effect recently discussed in Ref. 19. The model treats intercalation as a deposition process of neutral particles in the host lattice. In reality, however, one deals with the intercalation of positively charged ions while the negative ions are completely 
excluded from the host matrix. The exclusion of one ionic component from the host costs energy due to electrostatic attraction between co-ions and counterions. This effect modifies the energetic and entropic contributions to the free energy and destroys the particle-hole symmetry of the original lattice gas model.

\section{CONCLUSIONS}

A model of an intercalation compound proposed in the present paper takes into account the short-range electromagnetically mediated repulsive interaction between intercalants and their long-range attraction resulting from the strain field of the host matrix due to its distortion. The model predicts the basic features of the phase diagram which qualitatively explain the OCV of intercalation cells. As a future development of the theory we are planning to take into account the permselectivity effect and discuss other scenarios of dilation of the host material.

\section{ACKNOWLEDGMENTS}

The authors are grateful to J. R. Heringa and H. W. J. Blöte for fruitful discussions.

${ }^{1}$ W. R. McKinnon, in Nanomaterials: Synthesis, Properties, and Applications, edited by A. Edelstein and R. C. Camarada (IOP, Bristol, 1996).

${ }^{2}$ A. R. Armstrong and P. G. Bruce, Nature (London) 381, 499 (1996); A. D. Robertson, A. R. West, and A. G. Ritchie, Solid State Ionics 104, 1 (1997);
M. K. Aydinol, A. F. Kohan, G. Ceder, K. Cho, and J. Joannopoulus, Phys. Rev. B 56, 1354 (1997); C. Wolverton and A. Zunger, ibid. 57, 2242 (1998).

${ }^{3}$ Q. Zhong, J. R. Dahn, and K. Colbow, Phys. Rev. B 46, 2554 (1992).

${ }^{4}$ Y. Gao, J. N. Reimers, and J. R. Dahn, Phys. Rev. B 54, 3878 (1996).

${ }^{5}$ M. S. Whittingham, J. Electrochem. Soc. 123, 315 (1976).

${ }^{6}$ D. G. Clerc, R. D. Poshusta, and A. C. Hess, J. Phys. Chem. A 101, 8926 (1997).

${ }^{7}$ H. Abiko, M. Hibino, and T. Kudo, Electrochem. Solid-State Lett. 1, 114 (1998).

${ }^{8}$ G. Alefeld, Ber. Bunsenges. Phys. Chem. 76, 746 (1972).

${ }^{9}$ W. R. McKinnon and R. R. Haering, Solid State Ionics 1, 111 (1980).

${ }^{10}$ A. Mällo and U. Yxklinten, Z. Phys. B: Condens. Matter 83, 216 (1991).

${ }^{11}$ G. Leibfried and N. Breuer, Point Defects in Metals I. Introduction to the Theory, Vol. 81, in Springer Tracts in Modern Physics (Springer, New York, 1978).

${ }^{12}$ L. D. Landau and E. M. Lifshitz, Elasticity Theory (Pergamon, Oxford, 1959).

${ }^{13}$ H. Wagner and H. Horner, Adv. Phys. 23, 587 (1974).

${ }^{14}$ V. I. Kalikmanov, M. V. Koudriachova, and S. W. de Leeuw, Solid State Ionics 127, 163 (2000).

${ }^{15}$ R. J. Baxter, Exactly Solved Models in Statistical Mechanics (Academic, London, 1982).

${ }^{16}$ According to the definition (13), $\xi^{\mathrm{MF}}$ is a dimensionless material parameter; so different $\xi^{\mathrm{MF}}$ correspond to different compounds.

${ }^{17}$ W. Liu, K. Kowal, and G. C. Farrington, J. Electrochem. Soc. 145, 459 (1998).

${ }^{18}$ Note, that on the mean-field level the 3D diamond lattice and the 2D square lattice are indistinguishable (for both of them $q=4$ ); however, the "exact" solutions for them will be different.

${ }^{19}$ E. V. Vakarin et al., Phys. Rev. B 63, 014304 (2000); Vorotyntsev and J. P. Badiali, Electrochim. Acta 39, 289 (1994). 